\title{
Existence of positive solutions to discrete second- order boundary value problems with indefinite weight
}

\author{
Chenghua Gao*, Guowei Dai and Ruyun Ma
}

\author{
* Correspondence: gaokuguo@163. \\ com \\ Department of Mathematics, \\ Northwest Normal University, \\ Lanzhou 730070, P. R. China
}

\author{
Abstract \\ Let $T>1$ be an integer, $\mathbb{Z}=\{1,2, \ldots, T\}$. This article is concerned with the global \\ structure of the set of positive solutions to the discrete second-order boundary value \\ problems

$$
\begin{gathered}
\Delta^{2} u(t-1)+r m(t) f(u(t))=0, \quad t \in \mathbb{T}, \\
u(0)=u(T+1)=0,
\end{gathered}
$$ \\ where $r \neq 0$ is a parameter, $m: \mathbb{T} \rightarrow \mathbb{R}$ changes its sign, $m(t) \neq 0$ for $t \in \mathbb{T}$ and $f: \mathbb{R}$ \\ $\rightarrow \mathbb{R}$ is continuous. Also, we obtain the existence of two principal eigenvalues of the \\ corresponding linear eigenvalue problems. \\ MSC (2010): 39A12; 34B18.
}

Keywords: discrete indefinite weighted problems, positive solutions, principal eigenvalue, bifurcation, existence

\section{Introduction}

Let $T>1$ be an integer, $\mathbb{T}=\{1,2, \ldots, T\}$. This article is concerned with the global structure of the set of positive solutions to the discrete second-order boundary value problem (BVP)

$$
\begin{aligned}
& \Delta^{2} u(t-1)+r m(t) f(u(t))=0, \quad t \in \mathbb{T}, \\
& u(0)=u(T+1)=0,
\end{aligned}
$$

where $r \neq 0$ is a parameter, $f: \mathbb{R} \rightarrow \mathbb{R}$ is continuous, $m(t) \neq 0$ for $t \in \mathbb{T}$ and $m: \mathbb{T} \rightarrow \mathbb{R}$ changes its sign, i.e., there exists a proper subset $\mathbb{T}_{+}$of $\mathbb{T}$, such that $m(t)>0$ for $t \in \mathbb{T}_{+}$and $m(t)<0$ for $t \in \mathbb{T} \backslash \mathbb{T}_{+}$.

BVPs with indefinite weight arise from a selection-migration model in population genetics, see Fleming [1]. That an allele $A_{1}$ holds an advantage over a rival allele $A_{2}$ at some points and holds an disadvantage over $A_{2}$ at some other points can be presented by changing signs of $m$. The parameter $r$ corresponds to the reciprocal of the diffusion. The existence and multiplicity of positive solutions of BVPs for second-order differential equations with indefinite weight has been studied by many authors, see, for example [2-5] and the references therein. In [2], using Crandall-Rabinowitz's Theorem and Rabinowitz's global bifurcation theorem, Delgado and Suárez obtained the existence 
and multiplicity of positive solutions under Dirichlet boundary value condition. In 2006, Afrouzi and Brown [3] also obtained the similar results by using the mountain pass theorem. When $f$ is concave-convex type, similar results were also obtained by Ma and Han [4] and Ma et al. [5], and the main tool they used was the Rabinowitz's global bifurcation theorem.

For the discrete case, there is much literature dealing with different equations similar to (1.1) subject to various boundary value conditions. We refer to [6-14] and the reference therein. In particular, when $m(t)>0$ on $\mathbb{T}$, fixed point theorems, the discrete Gelfand theorem and the bifurcation techniques have been used to discuss the existence of positive solutions to the discrete problems, see, for example [6-8,12-14]. However, there are few results on the existence of positive solutions of (1.1) and (1.2) when $m(t)$ changes its sign on $\mathbb{T}$. Maybe the main reason is that the spectrum of the following linear eigenvalue problems

$$
\begin{aligned}
& \Delta^{2} u(t-1)+\lambda m(t) u(t)=0, \quad t \in \mathbb{T}, \\
& u(0)=u(T+1)=0
\end{aligned}
$$

is not clear when $m$ changes its sign on $\mathbb{T}$.

It is another aim of our article to give some information of the spectrum of (1.3) and (1.4). In this article, we will show that (1.3) and (1.4) has two principal eigenvalues $\lambda_{m \text {,- }}$ $<0<\lambda_{m,+}$, and the corresponding eigenfunctions which we denote by $\psi_{m,-}$ and $\psi_{m,+}$ don't change their signs on $\mathbb{T}$. Based on this result, using Rabinowitz's global bifurcation theorem [15], we will discuss the global structure of the set of positive solutions of (1.1), (1.2), and obtain the existence of positive solutions of (1.1) and (1.2). Moreover, we can also obtain the existence of negative solutions of (1.1) and (1.2).

Now, we give the definition of a positive solution and a negative solution of (1.1) and (1.2).

Definition 1.1. A positive solution of problem (1.1) and (1.2) refers to a pair $(r, u)$, where $r \neq 0, u$ is a solution of (1.1) with $u>0$ on Tand $u$ satisfies (1.2). Meanwhilem $(r, u)$ is called a negative solution of (1.1) and (1.2), if $(r,-u)$ is a positive solution of (1.1) and (1.2).

The article is arranged as follows. In Section 2, we state the Rabinowitz's global bifurcation theorem. In Section 3, the existence of two principal eigenvalues of (1.3) and (1.4) will be discussed. In Section 4, we state the main result and provide the proof.

\section{Preliminaries}

For the readers' convenience, we state the Rabinowitz's global bifurcation theorem [15] here.

Let $E$ be a real Banach space. Consider the equation

$$
u=\lambda T u+H(\lambda, u),
$$

which possesses the line of solutions $\{(\lambda, 0) \mid \lambda \in \mathbb{R}\}$ henceforth referred to as the trivial solutions, where $T: E \rightarrow E$ is a bounded linear operator and $H(\lambda, u)$ is continuous on $\mathbb{R} \times E$ with $H(\lambda, u)=o(\Downarrow u \bigotimes)$ near $u=0$ uniformly on bounded $\lambda$ intervals. 
Moreover, we assume that $T$ and $H$ are compact on $E$ and $\mathbb{R} \times E$, respectively, i.e., are continuous and they map bounded sets into relatively compact sets.

we will say $\mu$ is a characteristic value of $T$ if there exists $v \in E, v \neq 0$, such that $v=$ $\mu T \nu$, i.e., $\mu^{-1}$ is a nonzero eigenvalue of $T$. Let $r(T)$ denote the set of real characteristic values of $T$ and $\Gamma$ denote the closure of the set of nontrivial solutions of (2.1).

Theorem 2.1 ([15, Theorem 1.3]). If $\mu \in r(T)$ is of odd multiplicity, then $\Gamma$ contains a maximum subcontinuum $\mathcal{C}$ such that $(\mu, 0) \in \mathcal{C}$ and either

(i) meets $\infty$ in $\mathbb{R} \times E$,

or

(ii) meets $(\tilde{\mu}, 0)$ where $\mu \neq \tilde{\mu} \in r(T)$.

From [15], there exist two connected subsets, $\mathcal{C}^{+}$and $\mathcal{C}^{-}$, of $\mathcal{C}$ such that $\mathcal{C}=\mathcal{C}^{+} \cup \mathcal{C}^{-}$ and $\mathcal{C}^{+} \cap \mathcal{C}^{-}=\{(\mu, 0)\}$. Furthermore, Rabinowitz also shows that

Theorem 2.2 ([15, Theorem 1.40]). Each of $\mathcal{C}^{+}, \mathcal{C}^{-}$meets $(\mu, 0)$ and either

(i) meets $\infty$ in $\mathbb{R} \times E$,

or

(ii) meets $(\tilde{\mu}, 0)$ where $\mu \neq \tilde{\mu} \in r(T)$.

\section{Existence of two principal eigenvalues to (1.3) and (1.4)}

Recall that $\mathbb{T}=\{1,2, \ldots, T\}$. Let $\hat{\mathbb{T}}=\{0,1, \ldots, T+1\}$. Let $X=\{u: \hat{\mathbb{T}} \rightarrow \mathbb{R} \mid u(0)=u(T+1)=0\}$. Then $X$ is a Banach space under the norm $\|u\|_{X}=\max _{t \in \hat{\mathbb{T}}}|u(t)|$. Let $Y=\{u \mid u: \mathbb{T} \rightarrow \mathbb{R}\}$. Then $Y$ is a Banach space under the norm $\|u\|_{Y}=\max _{t \in \mathrm{T}}|u(t)|$.

Define the operator $L: X \rightarrow Y$ by

$$
L u(t)=-\Delta^{2} u(t-1), \quad t \in \mathbb{T} .
$$

In this section, we will discuss the existence of principal eigenvalues for the BVP (1.3) and (1.4). At first, we give the definition of principal eigenvalue of (1.3) and (1.4).

Definition 3.1. An eigenvalue $\lambda$ for (1.3) and (1.4) is called principal if there exists a nonnegative eigenfunction corresponding to $\lambda$, i.e., if there exists a nonnegative $u \in X \backslash$ $\{0\}$ such that $(\lambda, u)$ is a solution of (1.3) and (1.4).

The main idea we will use arises from [16,17]. For the reader's convenience, we state them here. At first, it is necessary to provide the definition of simple eigenvalue.

Definition 3.2. An eigenvalue $\lambda$ of (1.3) and (1.4) is called simple if dim $\bigcup_{n=1}^{\infty} \operatorname{ker}\left(I-\lambda L^{-1}\right)^{n}=1$, where kerA denotes the kernel of $A$.

Theorem 3.1. (1.3) and (1.4) has two simple principal eigenvalues.

Proof. Consider, for fixed $\lambda$, the eigenvalue problems

$$
L u-\lambda m(t) u(t)=\mu u(t), \quad t \in \mathbb{T},
$$




$$
u(0)=u(T+1)=0
$$

By Kelley and Peterson [18, Theorem 7.6], for fixed $\lambda$, (3.1), and (3.2) has $T$ simple eigenvalues

$$
\mu_{m, 1}(\lambda)<\mu_{m, 2}(\lambda)<\cdots<\mu_{m, T}(\lambda),
$$

and the corresponding eigenfunction $\psi_{m, k}(\lambda, t)$ has exactly $k-1$ simple generalized zeros.

Thus, $\lambda$ is a principal eigenvalue of (1.3) and (1.4), if and only if $\mu_{m, 1}(\lambda)=0$.

On the other hand, let

$$
S_{m, \lambda}=\left\{\sum_{t=0}^{T}|\Delta \phi(t)|^{2}-\lambda \sum_{t=1}^{T} m(t) \phi(t)^{2}: \phi \in X, \sum_{t=1}^{T} \phi(t)^{2}=1\right\} .
$$

Clearly, $S_{m, \lambda}$ is bounded below and $\mu_{m, 1}(\lambda)=\inf _{\varphi \in X} S_{m, \lambda}$, see [18, Theorem 7.7].

For fixed $\phi \in X, \lambda \rightarrow \sum_{t=0}^{T}|\Delta \phi(t)|^{2}-\lambda \sum_{t=1}^{T} m(t) \phi^{2}(t)$ is an affine function and so a concave function. As the infimum of any collection of concave functions is concave, it follows that $\lambda \rightarrow \mu_{m, 1}(\lambda)$ is a concave function. Also, by considering test functions $\varphi_{1}$, $\varphi_{2} \in X$ such that $\sum_{t=1}^{T} m(t) \phi_{1}^{2}(t)<0$ and $\sum_{t=1}^{T} m(t) \phi_{2}^{2}(t)>0$, it is easy to see that $\mu_{m, 1}(\lambda) \rightarrow-\infty$ as $\lambda \rightarrow \pm \infty$. Thus, $\lambda \rightarrow \mu_{m, 1}(\lambda)$ is an increasing function until it attains its maximum, and is a decreasing function thereafter.

Since $\mu_{m, 1}(0)>0, \lambda \rightarrow \mu_{m, 1}(\lambda)$ must have exactly two zeros. Thus, (1.3) and (1.4) has exactly two principal eigenvalues, $\lambda_{m,+}>0$ and $\lambda_{m,-}<0$, and the corresponding eigenfunctions don't change sign on $\hat{\mathbb{T}}$.

Now, we give a property for the above two principal eigenvalues.

Theorem 3.2. If $m, m_{1}: \mathbb{T} \rightarrow \mathbb{R}$ change their signs, and $m(t) \leq m_{1}(t)$ for $t \in \mathbb{T}$, then $\lambda_{m_{1},-} \leq \lambda_{m,-}, \lambda_{m_{1},+} \leq \lambda_{m,+}$

Proof. It can be seen that for $\lambda<0, S_{m, \lambda} \geq S_{m_{1}, \lambda}$, which implies $\mu_{m, 1}(\lambda) \geq \mu_{m_{1}, 1}(\lambda)$ and consequently, $\lambda_{m_{1+}} \geq \lambda_{m_{1,+}}$.

On the other hand, for $\lambda<0, S_{m, \lambda} \leq S_{m_{1}, \lambda}$, which indicates $\mu_{m, 1}(\lambda) \leq \mu_{m_{1}, 1}(\lambda)$ and consequently, $\lambda_{m,-} \geq \lambda_{m_{1},-}$.

\section{Main result}

We make the following assumptions.

(H1) $f: \mathbb{R} \rightarrow \mathbb{R}$ is continuous and $s f(s)>0$ for $s \neq 0$.

(H2) $f_{0}=\lim _{|s| \rightarrow 0} \frac{f(s)}{s} \in(0, \infty), \quad f_{\infty}=\lim _{|s| \rightarrow+\infty} \frac{f(s)}{s} \in(0, \infty)$.

Theorem 4.1. Suppose that (H1) and (H2) hold Assume that

$$
r \in\left(\frac{\lambda_{m,+}}{f_{\infty}}, \frac{\lambda_{m,+}}{f_{0}}\right) \cup\left(\frac{\lambda_{m,-}}{f_{0}}, \frac{\lambda_{m,-}}{f_{\infty}}\right),
$$

or

$$
r \in\left(\frac{\lambda_{m,+}}{f_{0}}, \frac{\lambda_{m,+}}{f_{\infty}}\right) \cup\left(\frac{\lambda_{m,-}}{f_{\infty}}, \frac{\lambda_{m,-}}{f_{0}}\right)
$$


Then (1.1) and (1.2) has two solutions $u^{+}$and $u^{-}$such that $u^{+}$is positive on Tand $u^{-}$ is negative on $\mathbb{T}$.

Obviously, we can get the following lemma with ease.

Lemma 4.1. Suppose that $u \in X$ and $u \neq \equiv$ on Tsatisfies (1.1) (or (1.3)) and there exists $t_{0} \in$ Tsuch that $u\left(t_{0}\right)=0$, then $u\left(t_{0}-1\right) u\left(t_{0}+1\right)<0$.

Proof of Theorem 4.1. First, we deal with the case $r>0$.

Let $\zeta, \xi \in C(\mathbb{R}, \mathbb{R})$ such that

$$
f(u)=f_{0} u+\zeta(u), \quad f(u)=f_{\infty} u+\xi(u) .
$$

Clearly

$$
\lim _{|u| \rightarrow 0} \frac{\zeta(u)}{u}=0, \quad \lim _{|u| \rightarrow \infty} \frac{\xi(u)}{u}=0 .
$$

Let

$$
\tilde{\xi}(u)=\max _{0 \leq|s| \leq u}|\xi(s)| .
$$

Then $\tilde{\xi}$ is nondecreasing and

$$
\lim _{|u| \rightarrow \infty} \frac{\tilde{\xi}(u)}{u}=0
$$

Let us consider

$$
L u-\lambda m(t) r f_{0} u-\lambda m(t) r \zeta(u)=0,
$$

as a bifurcation problem from the trivial solution $u \equiv 0$.

Equation (4.5) can be converted to the equivalent equation

$$
\begin{aligned}
u(t) & =\lambda L^{-1}\left[m(\cdot) r f_{0} u(\cdot)+m(\cdot) r \zeta(u(\cdot))\right](t) \\
& :=\lambda T u+H(\lambda, u) .
\end{aligned}
$$

It is easy to see that $T: X \rightarrow X$ is compact. Further we note that $H(\lambda, u)=\lambda L^{-1}[m$ $(\cdot) \zeta(u(\cdot))]=o(\bigotimes u \bigotimes)$ near $\lambda=0$ uniformly on bounded $\lambda$ intervals, since

$$
\begin{aligned}
\left\|L^{-1}[m(\cdot) \zeta(u(\cdot))]\right\| & =\max _{t \in \mathrm{T}}\left|\sum_{s=1}^{T} G(t, s) m(s) \zeta(u(s))\right| \\
& \leq C \cdot \max _{s \in \mathrm{T}}|m(s)\|\mid \zeta(u(\cdot))\|,
\end{aligned}
$$

where $C=\max _{t \in \hat{T}} \sum_{s=1}^{T} G(t, s)$ and

$$
G(t, s)=\frac{1}{T+1}\left\{\begin{array}{l}
(T+1-t) s, 0 \leq s \leq t \leq T+1 \\
(T+1-t) t, 0 \leq t \leq s \leq T+1
\end{array}\right.
$$

Let $\mathbb{E}=\mathbb{R} \times X$ under the product topology. Let $S^{+}:=\{u \in X \mid u(t)>0$ for $t \in \mathbb{T}\}$. Set $S$ $=-S^{+}, S=S^{+} \cup S^{-}$. Then $S^{+}$and $S^{-}$are disjoint in $X$. Finally let $\Psi^{ \pm}=\mathbb{R} \times S^{ \pm}$and $\Psi=\mathbb{R}$ $\times S$. Let $\Sigma$ be the closure of the set of nontrivial solutions of (1.1) and (1.2).

It is easy to see that $\frac{\lambda_{m_{1}+}}{r f_{0}} \in r(T)$ is simple. Now applying Theorems 2.1 and 2.2, we get the result as follows: $\Sigma$ contains a maximum subcontinuum $\mathcal{C}_{+}$which is composed of two distinct connected set $\mathcal{C}_{+}^{+}$and $\mathcal{C}_{+}^{-}$such that $\mathcal{C}_{+}=\mathcal{C}_{+}^{+} \cup \mathcal{C}_{+}^{-}$and 
$\mathcal{C}_{+}^{+} \cap \mathcal{C}_{+}^{-}=\left\{\left(\frac{\lambda_{m,+}}{r f_{0}}, 0\right)\right\}$. Moreover, Lemma 4.1 guarantees the second case in Theorems 2.1 and 2.2 cannot happen. Otherwise, there will exist $(\eta, y) \in C_{+}^{v}$, such that $y$ has a multiple zero point $t_{0}$, (i.e., $t_{0}$ satisfies $y\left(t_{0}\right)=0$ and $y\left(t_{0}-1\right) y\left(t_{0}+1\right)>0$ ). However, this contradicts Lemma 4.1. Thus, for each $v \in\{+,-\}, \mathcal{C}_{+}^{v}$ joins $\left(\frac{\lambda_{m,+}}{r f_{0}}, 0\right)$ to infinity in $\Psi^{\nu}$ and $\mathcal{C}_{+}^{\nu} \backslash\left\{\left(\frac{\lambda_{m_{1}}}{r f_{0}}, 0\right)\right\} \subset \Psi^{\nu}$.

It is obvious that any solution to $(4.5)$ of the form $(1, u)$ yields a solution $u$ to $(1.1)$ and (1.2). We will show that $\mathcal{C}_{+}^{v}$ crosses the hyperplane $\{1\} \times X$ in $\mathbb{R} \times X$. To achieve this goal, it will be enough to show that

$$
\left(\frac{\lambda_{m,+}}{r f_{\infty}}, \frac{\lambda_{m,+}}{r f_{0}}\right) \subseteq \operatorname{Proj}_{\mathbb{R}} \mathcal{C}_{+}^{v}
$$

or

$$
\left(\frac{\lambda_{m,+}}{r f_{0}}, \frac{\lambda_{m,+}}{r f_{\infty}}\right) \subseteq \operatorname{Proj}_{\mathbb{R}} \mathcal{C}_{+}^{v}
$$

where Proj $j_{\mathbb{R}} \mathcal{C}_{+}^{v}$ denotes the projection of $\mathcal{C}_{+}^{v}$ on $\mathbb{R}$.

Let $\left(\mu_{n}, y_{n}\right) \in \mathcal{C}_{+}^{v}$ satisfy

$$
\mu_{n}+\left\|y_{n}\right\|_{X} \rightarrow \infty \text {. }
$$

We note that $\mu_{n}>0$ for all $n \in \mathbb{N}$ since $(0,0)$ is the only solution of (4.5) for $\lambda=0$ and $\mathcal{C}_{+}^{v} \cap(\{0\} \times X)=\emptyset$.

Case 1. $\frac{\lambda_{m,+}}{r f_{\infty}}<1<\frac{\lambda_{m,+}}{r f_{0}}$.

We divide the proof into two steps.

Step 1. We show that if there exists a constant number $M>0$ such that

$$
\mu_{n} \in(0, M]
$$

then (4.7) holds.

In this case it follows that

$$
\left\|y_{n}\right\|_{X} \rightarrow \infty \text {. }
$$

We divide the equation

$$
L y_{n}-\mu_{n} m(t) r f_{\infty} y_{n}-\mu_{n} m(t) r \xi\left(y_{n}\right)=0
$$

by $₫ y_{n} \bigotimes_{x}$ and set $\bar{\gamma}_{n}=\frac{\gamma_{n}}{\left\|y_{n}\right\| x}$. Since $\bar{\gamma}_{n}$ is bounded in $X$ and $\mu_{n}$ is bounded in $\mathbb{R}$, after taking the subsequence if necessary, we have that $\bar{y}_{n} \rightarrow \bar{y}$ for some $\bar{y} \in X$ with $\|\bar{y}\|_{X}=1$ and $\mu_{n} \rightarrow \bar{\mu}$ for some $\mu \in \mathbb{R}$. Moreover, from (4.4) and the fact that $\tilde{\xi}$ is nondecreasing, we have that

$$
\lim _{n \rightarrow \infty} \frac{\left|\xi\left(y_{n}(t)\right)\right|}{\left\|y_{n}\right\|_{X}}=0
$$




$$
\begin{gathered}
\text { since } \frac{\left|\xi\left(y_{n}(t)\right)\right|}{\left\|y_{n}\right\|_{X}} \leq \frac{\tilde{\xi}\left(\left|y_{n}(t)\right|\right)}{\left\|y_{n}\right\|_{X}} \leq \frac{\xi\left(\left\|y_{n}\right\|_{X}\right)}{\left\|y_{n}\right\|_{X}} \text {. Thus, } \\
\bar{\gamma}(t)=\sum_{s=1}^{T} G(t, s) \bar{\mu} m(s) r f_{\infty} \bar{\gamma}(s)
\end{gathered}
$$

which implies that

$$
L \bar{y}-\bar{\mu} m(t) r f_{\infty} \bar{\gamma}=0 .
$$

We claim that

$$
(\bar{\mu}, \bar{y}) \in \mathcal{C}_{+}^{v} .
$$

We only prove that if $y_{n} \in \mathcal{C}_{+}^{+}$, then $\bar{y}_{n} \in \mathcal{C}_{+}^{+}$. The other case that if $y_{n} \in \mathcal{C}_{+}^{-}$, then $\bar{y}_{n} \in \mathcal{C}_{+}^{-}$can be treated similarly.

Obviously when $y_{n} \in \mathcal{C}_{+}^{+}$, then $\bar{y}(t) \geq 0$ on $\hat{\mathbb{T}}$. Furthermore, $\bar{\gamma}(t)>0$ on $\mathbb{T}$. In fact, if there exists a $t_{0} \in \mathbb{T}$ such that $\bar{y}\left(t_{0}\right)=0$, then, by Lemma 4.1, we obtain $\bar{\gamma}\left(t_{0}-1\right) \bar{\gamma}\left(t_{0}+1\right)<0$ which contradicts the fact that $\bar{\gamma}(t) \geq 0$ on $\hat{\mathbb{T}}$. Thus, $\bar{\gamma}(t)>0$ on $\mathbb{T}$. This together with the fact $\mathcal{C}_{+}$is a closed set in $\mathbb{E}$ implies that $\bar{y} \in \mathcal{C}_{+}^{+}$. Moreover, $\bar{\mu} r f_{\infty}=\lambda_{m,+}$, so that

$$
\bar{\mu}=\frac{\lambda_{m,+}}{r f_{\infty}} .
$$

Thus, (4.7) holds.

Step 2. We show that there exists a constant $M>0$ such that $\mu_{n} \in(0, M]$ for all $n$. Since $\left\{\left(\mu_{n}, y_{n}\right)\right\}$ are the solutions to (4.5), they follow that

$$
L y_{n}=\mu_{n} r m(t) \Gamma_{n}(t) y_{n}
$$

where $\Gamma_{n}(t):=\frac{f\left(y_{n}(t)\right)}{\gamma_{n}(t)}$. From $(\mathrm{H} 1)$ and $(\mathrm{H} 2)$, there exist two positive constants $\rho_{1}$ and $\rho_{2}$, such that

$$
\rho_{1}<\frac{f\left(y_{n}\right)}{y_{n}}<\rho_{2}
$$

Let $\eta_{*}>0$ be the positive principal eigenvalue of the following linear eigenvalue problem

$$
L v=\eta \chi_{1}(t) m(t) v
$$

and $\eta^{*}>0$ the positive principal eigenvalue of the following linear eigenvalue problem

$$
L v=\eta \chi_{2}(t) m(t) v,
$$

where

$$
\chi_{1}(t)=\left\{\begin{array}{l}
\rho_{1} \text { if } m(t)>0, t \in \mathbb{T}, \\
\rho_{2} \text { if } m(t)<0, t \in \mathbb{T},
\end{array} \chi_{2}(t)=\left\{\begin{array}{l}
\rho_{2} \text { if } m(t)>0, t \in \mathbb{T}, \\
\rho_{1} \text { if } m(t)<0, t \in \mathbb{T} .
\end{array}\right.\right.
$$


By Theorem 3.2, (4.14), (4.15), (4.16), and (4.17), we get

$$
\frac{\eta^{*}}{r}<\mu_{n}<\frac{\eta_{*}}{r} .
$$

Case 2. $\frac{\lambda_{m,+}}{r f_{0}}<1<\frac{\lambda_{m,+}}{r f_{\infty}}$.

From Step 2 of Case 1, there exists $M>0$ such that for all $n \in \mathbb{N}$,

$$
\mu_{n} \in(0, M]
$$

Applying a similar argument to that used in Step 1 of Case 1 (after taking a subsequence and relabeling, if necessary), we get

$$
\mu_{n} \rightarrow \frac{\lambda_{m,+}}{r f_{\infty}}, \quad y_{n} \rightarrow \infty \quad \text { as } n \rightarrow \infty
$$

which implies that (4.8) holds.

At last, we deal with the case $r<0$.

Let us consider

$$
L u-\lambda r m(t) f_{0} u-\lambda r m(t) \zeta(u)=0,
$$

as a bifurcation problem from the trivial solution $u \equiv 0$. Now, applying Theorems 2.1 and 2.2, we get the following results: $\Sigma$ contains a maximum subcontinuum $\mathcal{C}_{-}$which is composed of two distinct connected set $\mathcal{C}_{-}^{+}$and $\mathcal{C}_{-}^{-}$such that $\mathcal{C}_{-}=\mathcal{C}_{-}^{+} \cup \mathcal{C}_{-}^{-}$and $\mathcal{C}_{+}^{-} \cap \mathcal{C}_{-}^{-}=\left\{\left(\frac{\lambda_{m,-}}{-r f_{0}}, 0\right)\right\}$. Moreover, by Lemma 4.1, for each $v \in\{+,-\}, \mathcal{C}_{-}^{v}$ joins $\left(\frac{\lambda_{m,-}}{-r f_{0}}, 0\right)$ to infinity in $\Psi^{\nu}$ and $\mathcal{C}_{-}^{\nu} \backslash\left\{\left(\frac{\lambda_{m,}}{-r f_{0}}, 0\right)\right\} \subset \Psi^{\nu}$, where $\Sigma$ and $\Psi^{v}$ are defined as in the case $r>0$.

It is clear that any solution to (4.18) of the form $(-1, u)$ yields a solutions $u$ of (1.1) and (1.2). We will show $\mathcal{C}_{-}^{v}$ crosses the hyperplane $\{-1\} \times X$ in $\mathbb{R} \times X$. To achieve this goal, it will be enough to show that

$$
\left(\frac{\lambda_{m,-}}{-r f_{\infty}}, \frac{\lambda_{m,-}}{-r f_{0}}\right) \subseteq \operatorname{Proj}_{\mathbb{R}} \mathcal{C}_{-}^{v},
$$

or

$$
\left(\frac{\lambda_{m_{-}-}}{-r f_{0}}, \frac{\lambda_{m,-}}{-r f_{\infty}}\right) \subseteq \operatorname{Proj}_{\mathbb{R}} \mathcal{C}_{-}^{v}
$$

Let $\left(\mu_{n}, y_{n}\right) \in \mathcal{C}_{-}^{v}$ satisfy

$$
\left|\mu_{n}\right|+\left\|\gamma_{n}\right\|_{X} \rightarrow \infty \text {. }
$$

We note that $\mu_{n}<0$ for all $n \in \mathbb{N}$ since $(0,0)$ is the only solution to (4.18) for $\lambda=0$ and $\mathcal{C}_{-}^{v} \cap(\{0\} \times X)=\emptyset$.

The rest of the proof is similar to the proof of the case $r>0$, so we omit it.

\section{Example}

Let $T=5$, then $\mathbb{T}=\{1,2,3,4,5\}$. Consider the following discrete second-order BVPs

$$
\Delta^{2} u(t-1)+\lambda m(t) f(u(t))=0, \quad t \in \mathbb{T},
$$




$$
u(0)=u(6)=0,
$$

where $m: \mathbb{T} \rightarrow \mathbb{R}$ which is defined by

$$
m(1)=1, m(2)=2, m(3)=1, m(4)=-1, m(5)=-3,
$$

and

$$
f(s)=\frac{s^{3}+s}{s^{2}+2} .
$$

By using Matlab 7.0, we get the following eigenvalue problem

$$
\begin{aligned}
& \Delta^{2} u(t-1)+\lambda m(t) u(t)=0, \quad t \in \mathbb{T}, \\
& u(0)=u(6)=0
\end{aligned}
$$

has two principal eigenvalues $\lambda_{m,-}=-0.5099$ and $\lambda_{m,+}=0.2867$. The corresponding eigenfunctions

$\psi_{m,-}(t)$ and $\psi_{m,+}(t)$ satisfy

$$
\begin{gathered}
\psi_{m_{,}-}(0)=0, \psi_{m_{,}-}(1)=0.0471, \psi_{m_{1}-}(2)=0.1182 \\
\psi_{m,-}(3)=0.3099, \psi_{m_{,}-}(4)=0.6595, \psi_{m,-}(5)=0.6729, \psi_{m_{,}-}(6)=0
\end{gathered}
$$

and

$$
\begin{gathered}
\psi_{m_{,}+}(0)=0, \psi_{m_{,+}}(1)=0.3867, \psi_{m_{,+}}(2)=0.6626 \\
\psi_{m_{,+}}(3)=0.5584, \psi_{m_{,+}}(4)=0.2942, \psi_{m_{,}+}(5)=0.1143, \psi_{m_{,+}}(6)=0 .
\end{gathered}
$$

Moreover,

$$
\begin{gathered}
s f(s)=\frac{s^{4}+s^{2}}{s^{2}+2}>0, \quad \text { for } s \neq 0, \\
f_{0}=\lim _{|s| \rightarrow 0} \frac{f(s)}{s}=\lim _{s \rightarrow 0} \frac{s^{3}+s}{s^{3}+2 s}=\frac{1}{2} \in(0, \infty), \quad f_{\infty}=\lim _{|s| \rightarrow \infty} \frac{f(s)}{s}=\lim _{s \rightarrow \infty} \frac{s^{3}+s}{s^{3}+2 s}=1 \in(0, \infty) .
\end{gathered}
$$

Obviously, $f(s)$ satisfies (H1) and (H2). Thus, for

$$
r \in\left(\lambda_{m,+}, 2 \lambda_{m,+}\right)
$$

or

$$
r \in\left(2 \lambda_{m,-}, \lambda_{m,-}\right),
$$

(5.1) and (5.2) has a positive solution $u^{+}$and a negative solution $u^{-}$.

\section{Acknowledgements}

The authors were very grateful to the anonymous referees for their valuable suggestions. This research was supported by the National Natural Science Foundation of China (No. 11061030, 11101335,11126296) and the Fundamental Research Funds of the Gansu Universities.

\section{Authors' contributions}

The authors declare that the study was realized in collaboration with the same responsibility. All authors read and approved the final manuscript.

\section{Competing interests}

The authors declare that they have no competing interests.

Received: 14 August 2011 Accepted: 17 January 2012 Published: 17 January 2012 


\section{References}

1. Fleming, WH: A selection-migration model in population genetics. J Math Biol.2(1975), 219-233

2. Delgado, M, Suárez, A: On the existence and multiplicity of positive solutions for some indefinite nonlinear eigenvalue problem. Proc Amer Math Soc. 132(6), 1721-1728 (2004). doi:10.1090/S0002-9939-04-07233-8

3. Afrouzi, GA, Brown, KJ: Positive mountain pass solutions for a semilinear elliptic equation with a sign-changing weight function. Nonlinear Anal: TMA. 64, 409-416 (2006). doi:10.1016/j.na.2005.06.018

4. Ma, RY, Han, XL: Existence and multiplicity of positive solutions of a nonlinear eigenvalue problem with indefinite weight function. Appl Math Comput. 215, 1077-1083 (2009). doi:10.1016/j.amc.2009.06.042

5. $\mathrm{Ma}, \mathrm{RY}, \mathrm{Xu}, \mathrm{J}, \mathrm{Han}, \mathrm{XL}$ : Global bifurcation of positive solutions of a second-order periodic boundary value problems with indefinite weight. Nonlinear Anal: TMA. 71, 2119-2125 (2009). doi:10.1016/j.na.2009.01.046

6. Agarwal, RP, Henderson, J: Positive solutions and nonlinear eigenvalue problems for third order difference equations. Comput Math Appl. 36(10-12), 347-355 (1998). doi:10.1016/S0898-1221(98)80035-7

7. Agarwal, RP, O'Regan, D: Boundary value problems for discrete equations. Appl Math Lett. 10(4), 83-89 (1997). doi:10.1016/S0893-9659(97)00064-5

8. Atici, FM, Guseinov, GSh: On the existence of positive solutions for nonlinear differential equations with periodic boundary conditions. J Comput Appl Math. 132(2), 341-356 (2001). doi:10.1016/S0377-0427(00)00438-6

9. Rachunkova, I, Tisdell, CC: Existence of non-spurious solutions to discrete Dirichlet problems with lower and upper solutions. Nonlinear Anal: TMA. 67(4), 1236-1245 (2007). doi:10.1016/j.na.2006.07.010

10. Rodriguez, J: Nonlinear discrete Sturm-Liouville problems. J Math Anal Appl. 308(1), 380-391 (2005). doi:10.1016/j. jmaa.2005.01.032

11. Cheng, SS, Yen, HT: On a discrete nonlinear boundary value problem. Linear Algebra Appl. 313(1-3), 193-201 (2000). doi:10.1016/S0024-3795(00)00133-6

12. Zhang, G, Feng, W: On the number of positive solutions of a nonlinear algebraic system. Linear Algebra Appl. 422(2-3), 404-421 (2007). doi:10.1016/j.laa.2006.10.026

13. Ma, RY, Ma, HL: Positive solutions for nonlinear discrete periodic boundary value problems. Comput Math Appl. 59 , 136-141 (2010). doi:10.1016/j.camwa.2009.07.071

14. $\mathrm{Ma}, \mathrm{RY}, \mathrm{Xu}, \mathrm{YJ}, \mathrm{Gao}, \mathrm{CH}$ : A global description of the positive solutions of sublinear second-order discrete boundary value problems. Adv Diff Equ, Art ID 671625. 15 (2009)

15. Rabinowitz, PH: Some global results of nonlinear eigenvalue problems. J Funct Anal. 7, 487-513 (1971). doi:10.1016/ 0022-1236(71)90030-9

16. Hess, P, Kato, T: On some linear and nonlinear eigenvalue problems with indefinite weight function. Comm Part Diff Equ. 5, 999-1030 (1980). doi:10.1080/03605308008820162

17. Afrouzi, GA, Brown, KJ: On principal eigenvlaues for boundary value problems with indefinite weight and Robin boundary conditions. Proc Amer Math Soc. 127(1), 125-130 (1999). doi:10.1090/S0002-9939-99-04561-X

18. Kelly, W, Peterson, A: Difference Equations. An Introduction with Applications. Academic Press, New York (1990)

doi:10.1186/1687-1847-2012-3

Cite this article as: Gao et al:: Existence of positive solutions to discrete second-order boundary value problems with indefinite weight. Advances in Difference Equations 2012 2012:3.

\section{Submit your manuscript to a SpringerOpen ${ }^{\circ}$ journal and benefit from:}

- Convenient online submission

- Rigorous peer review

- Immediate publication on acceptance

- Open access: articles freely available online

- High visibility within the field

- Retaining the copyright to your article

Submit your next manuscript at $>$ springeropen.com 